\title{
Philosophiques
}

\section{Faute de frappe : Derrida dactylo}

\section{Katie Chenoweth}

Volume 47, numéro 2, automne 2020

\section{Derrida en cours}

URI : https://id.erudit.org/iderudit/1075127ar

DOI : https://doi.org/10.7202/1075127ar

Aller au sommaire du numéro

\section{Éditeur(s)}

Société de philosophie du Québec

\section{ISSN}

0316-2923 (imprimé)

1492-1391 (numérique)

Découvrir la revue

\section{Citer cet article}

Chenoweth, K. (2020). Faute de frappe : Derrida dactylo. Philosophiques, 47(2), 333-349. https://doi.org/10.7202/1075127ar

\section{Résumé de l'article}

" Je tape très vite, très mal, avec beaucoup de fautes ", confesse Jacques Derrida dans une entrevue tardive. Suivant cette idée, cet article propose que l'erreur typographique - habituellement perçue comme un simple " accident » devant être corrigé ou normalisé - puisse en fait être comprise comme le site opérant de lectures et de réflexions déconstructives. En analysant le manuscrit dactylographié du séminaire "Le fantôme de l'autre " (1984-1985) à partir duquel sont dérivés les textes Geschlecht II et Geschlecht III et en m'appuyant sur la suggestion provocatrice de Nietzsche selon laquelle la machine à écrire " collabore " en quelque sorte à la réflexion, j'examine l'omniprésence des fautes de frappe chez Derrida et son rapport particulier à la machine à écrire. Je me concentre plus précisément sur les lectures de Heidegger dans ce séminaire afin de situer la pensée derridienne de la frappe (du Schlag), c'est-à-dire du coup, de la marque, de l'empreinte, au coeur de tous les «types » (une autre des nombreuses significations du mot allemand " Geschlecht »). J’affirme ainsi que la faute de frappe dans ce séminaire doit être considérée comme une condition de possibilité de tout type et qu'elle révèle une différence significative entre Derrida et Heidegger.
Ce document est protégé par la loi sur le droit d'auteur. L'utilisation des services d’Érudit (y compris la reproduction) est assujettie à sa politique d'utilisation que vous pouvez consulter en ligne.

https://apropos.erudit.org/fr/usagers/politique-dutilisation/ 


\title{
Faute de frappe: Derrida dactylo
}

\author{
KATIE CHENOWETH
}

Département de français et d'italien, Princeton University

\begin{abstract}
RÉSUMÉ. - «Je tape très vite, très mal, avec beaucoup de fautes », confesse Jacques Derrida dans une entrevue tardive. Suivant cette idée, cet article propose que l'erreur typographique - habituellement perçue comme un simple «accident» devant être corrigé ou normalisé — puisse en fait être comprise comme le site opérant de lectures et de réflexions déconstructives. En analysant le manuscrit dactylographié du séminaire «Le fantôme de l'autre» (1984-1985) à partir duquel sont dérivés les textes Geschlecht II et Geschlecht III et en m'appuyant sur la suggestion provocatrice de Nietzsche selon laquelle la machine à écrire «collabore» en quelque sorte à la réflexion, j'examine l'omniprésence des fautes de frappe chez Derrida et son rapport particulier à la machine à écrire. Je me concentre plus précisément sur les lectures de Heidegger dans ce séminaire afin de situer la pensée derridienne de la frappe (du Schlag), c'est-à-dire du coup, de la marque, de l'empreinte, au cœur de tous les «types» (une autre des nombreuses significations du mot allemand « Geschlecht»). J'affirme ainsi que la faute de frappe dans ce séminaire doit être considérée comme une condition de possibilité de tout type et qu'elle révèle une différence significative entre Derrida et Heidegger.
\end{abstract}

\begin{abstract}
I type very quickly, very badly, with many errors [fautes]," Jacques Derrida confessed in a late interview. This paper proposes that the typographical error - usually viewed as a mere "accident" to be corrected or normalized - may in fact be understood as a productive site for deconstructive reading and thought. Drawing on Nietzsche's provocative suggestion that the typewriter acts as a "collaborator" in thinking, I examine Derrida's use of the writing machine with an eye to his ubiquitous typos or fautes de frappe. I focus on Derrida's readings of Heidegger in the Geschlecht series, analyzing the typescript of Derrida's 1984-85 seminar "Le fantôme de l'autre" from which the texts of Geschlecht II and Geschlecht III are derived, in order to locate a Derridean thinking of the frappe or Schlag (that is, the blow, strike, or imprint) at the heart of every "type" (one of the many meanings of the German word "Geschlecht"). Following Derrida's reading in the Geschlecht series and his own typographical practice, I argue that the typo should be thought of as the condition of possibility of every type — and as a defining difference between Derrida and Heidegger.
\end{abstract}

Unser Schreibzeug arbeitet mit an unseren Gedanken ${ }^{1}$. FRIEDRICH NiETZSCHE, «Lettre à Heinrich Köselitz », 1882.

1. Friedrich Nietzsche, «An Heinrich Köselitz in Venedig (Typoskript)» [vers fin février 1882], dans Nietzsche Briefwechsel, Kritische Gesamtausgabe, III-1, Giorgio Colli et Mazzino Montinari (éds), Berlin, Walter de Gruyter, 1981, p. 172; "À Heinrich Köselitz à Venise (à la machine) ", trad. fr. Jean Lacoste, dans Correspondance IV (1880-1884), G. Colli et M. Montinari (éds), Paris, Gallimard, 2015, p. 177: «[...] notre outillage scripturaire forge avec nous nos pensées". 
Je tape très vite, très mal, avec beaucoup de fautes ${ }^{2}$. JACQUes DeRridA, «Entre le corps écrivant et l'écriture... », 2001.

Le premier des deux exergues que j'ai placés plus haut nous vient des toutes premières heures de la machine à écrire. Dans une lettre à Friedrich Nietzsche datée du 19 février 1882, l'auteur et compositeur allemand Heinrich Köselitz suggère que la nouvelle "machine à écrire » (Schreibapparat) de son ami laquelle lui avait été récemment offerte en cadeau par sa mère et sa sœur — pourrait bien avoir un effet sur sa pensée. "J'aimerais tant voir comment les manipulations interviennent par cette machine à écrire ${ }^{3}$ ", écrit Körselitz de la sphère à écrire de Nietzsche, la première machine inventée et commercialisée par le révérend Malling Hansen.

Je pense que cela prend beaucoup de pratique pour garder les lignes droites et fluides. Peut-être que vous allez adopter un nouvel idiome [eine nene Ausdrucksweise] avec cet instrument; ce serait du moins ce qui se produirait avec moi. Je ne cache pas que mes "pensées» sur la musique et le langage sont souvent dépendantes de la qualité de ma plume et du papier ${ }^{4}$.

Et plusieurs jours plus tard, Nietzsche répond à son ami: "Vous avez raison: notre outillage scripturaire forge avec nous [arbeitet mit an] nos pensées ${ }^{5}$.» Sommes-nous surpris que cette assertion "si scandaleuse aux yeux de la philosophie ${ }^{6}$ ", proposant que la machine à écrire soit une collaboratrice, qu'elle concoure au travail philosophique, ait été elle-même écrite sur — ou mieux, avec - une machine à écrire ${ }^{7}$ ? C'est avec sa machine à écrire que Nietzsche donne ainsi raison à son ami, tapant chaque lettre une à une: oui, la machine à écrire s'imprime sur nos pensées, elle y laisse une trace, une empreinte; oui, la machine pourrait être en mesure de produire un nouvel idiome philosophique (Ausdrucksweise). Et elle pourrait bien, cette machine, souligner cet idiome, l'exprimer au sens fort de ce terme, lorsqu'elle résiste à nos doigts, c'est-à-dire là où elle manque et où elle nous fait hésiter. "Quand mes doigts seront-ils capables, se demande Nietzsche, de taper [drücken] une longue phrase ${ }^{8}$ ?» Aujourd'hui, les effets de la machine sur la

2. Jacques Derrida, «Entre le corps écrivant et l'écriture...", entretien avec Daniel Ferrer, Genesis, n 17, 2001, p. 63.

3. Heinrich Köselitz, «Heinrich Köselitz an Nietzsche in Genua (Postkarte)» [19 février 1882], dans Nietzsche Briefwechsel, Kritische Gesamtausgabe, III-2, p. 229. Notre traduction.

4. Ibid.

5. Nietzsche, "À Heinrich Köselitz à Venise ", p. 177.

6. Friedrich Kittler, Gramophone, Film, Typewriter, trad. fr., Frédérique Vargoz, Dijon, Les Presses du réel, 2018, p. 334.

7. Comme la première édition des correspondances de Friedrich Nietzsche le note, il s'agit de la deuxième lettre à être écrite «mit der Schreibmaschine» (avec la machine à écrire), la première étant une série d'aphorismes datés du 17 février 1882 envoyés à Köselitz. C'est celleci qui incita ce dernier à réfléchir aux effets stylistiques occasionnés par la machine à écrire.

8. Nietzsche, "À Heinrich Köselitz à Venise », p. 177. 
production philosophique du philosophe sont bien connus: «Nietzsche [...] passa des arguments aux aphorismes, des pensées aux jeux de mots, de la rhétorique au style télégraphique. [...] La machine sphérique de Malling Hansen et ses difficultés d'utilisation rendirent Nietzsche laconique9. »

Or Nietzsche ne fut pas seulement le premier philosophe à écrire avec une machine, il fut aussi, en tant que l'un des tout premiers Allemands à posséder ce type d'appareil, ce que nous appellerions maintenant en anglais un early adopter. Au crépuscule de la métaphysique, Nietzsche fut ainsi un précurseur en adoptant une machine qui participe à l'élaboration de sa pensée. Au bas de sa lettre à Köselitz, il ajouta même un postscriptum à l'intention de son ami - cette fois-ci au crayon, de sa propre main - qui témoigne de sa position à l'aube des développements technomédiatiques qui suivront: «Diable! Vous pouvez déchiffrer cela auss ${ }^{10}$ ?" Cette machine que Nietzsche utilise donc rapidement est aussi la première à soutirer la typographie aux imprimeurs - et donc aussi la possibilité des erreurs typographiques, des fautes de frappe - en la déplaçant dans les mains de l'écrivain-penseur. Avant l'invention de la machine à écrire, l'erreur ou la coquille (la typo comme on dit en anglais) était exclusivement la faute du typographe ou du compositeur qui composait les caractères dans son atelier, ligne par ligne, une lettre à la fois. Depuis le $\mathrm{Xv}^{\mathrm{e}}$ siècle, l'erreur dans les impressions avait par conséquent été l'affaire des artisans et des spécialistes. Mais voilà, ce que fait la machine à écrire entre tant d'autres choses, c'est d'abord de démocratiser et de disséminer l'erreur typographique. Elle ramène à la portée de tous la faute (de frappe), que nous avons maintenant sous la main, au bout des doigts. En effet, ce n'est pas un hasard si le terme "typo », en anglais, n'est forgé, par abréviation du syntagme "typographical error ", qu'en 1891, apparaissant lui-même de la conjonction d'une économie de la machine ("Quand mes doigts seront-ils capables de taper une longue phrase ${ }^{11}$ ! $)$ et de l'accès nouveau à l'erreur dont il est ici question. La machine à écrire s'avère ainsi génératrice et d'aphorismes et de fautes. Celle de Nietzsche n'a pas fait exception. Bien qu'il ait ultimement abandonné sa sphère à écrire de Malling Hansen, ce qui nous reste de ses lettres et de ses nombreux vers dactylographiés demeure "tout au long entaché d'erreurs typographiques ${ }^{12}$; une page tirée des archives de Nietzsche se lit par exemple comme un déferlement débridé de fautes, s'entraînant l'une l'autre dans une symphonie illisible d'accidents en majuscules: "MELSDNDRGILSTHCZMQNMOY».

9. Kittler, Gramophone, Film, Typewriter, p. 338.

10. Nietzsche, "À Heinrich Köselitz à Venise ", p. 177. L'italique est dans le texte.

11. Ibid.

12. Christian J. Emden, Nietzsche on Language, Consciousness, and the Body, Champaign (IL), University of Illinois Press, 2010, p. 28. [Nous traduisons: « marred with typographical errors throughout» (NdT)]. 
Le second épigraphe nous catapulte quant à lui plus d'un siècle en avant, dans une ère technomédiatique où la machine à écrire a presque disparu. En 2001, Jacques Derrida donna une entrevue à l'École normale supérieure (ENS) dans laquelle il accepta de discuter dans un registre autobiographique, en long et en large, de ses propres pratiques de lecture et d'écriture. C'est dans celle-ci qu'il confesse: «Je tape très vite, très mal, avec beaucoup de fautes ${ }^{13}$. " Je propose d'aborder dans cet article, un phénomène qui, à ma connaissance, n'a pas été reconnu, ou trop rarement, dans les études derridiennes (peut-être parce que cela risque de sembler anecdotique ou trop biographique, du moins cela court le risque de ne pas apparaître comme un sujet sérieux ou proprement philosophique), c'est-à-dire le fait que lorsqu'il tape - comme il l'a fait à partir de la fin des années 1960 pour tous ses séminaires et tous ses textes - Derrida multiplie les fautes typographiques, ce qu'il admet volontiers. Les archives de Derrida, y compris le tapuscrit du séminaire 1984-1985 duquel sont tirés les textes de Geschlecht II et Geschlecht III, souffrent de nombreuses erreurs. Je propose donc de lire ici, dans la série des Geschlechter, les fautes de frappe (expression qui en français évoque littéralement une erreur dans le mouvement d'actionner avec les doigts, de taper ou de frapper, les touches). J'entreprendrai donc cette lecture dans l'objectif de penser la faute typographique comme condi-

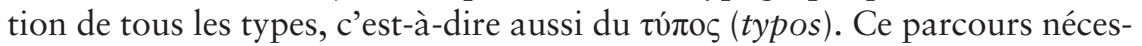
sitera, entre autres, de prendre au sérieux l'aphorisme nietzschéen ("Unser Schreibzeug arbeitet mit an unseren Gedanken ") et ce qu'il suggère - scandaleusement, peut-être — quant à la relation entre la pensée et le Schreibzeng, à savoir les outils, le matériel, les instruments ou l'équipement d'écriture.

Ce même mot allemand (Schreibzeug) se trouve en effet dans la discussion heideggérienne de l'«être-sous-la-main » au $\mathbb{\$} 15$ d'Être et Temps. Là, il ne désigne pas la machine à écrire, mais plutôt le Schreibzeug préféré de Heidegger: "la plume, l'encre, le papier, le sous-main ${ }^{14}$ ». Le Schreibzeug fait aussi une apparition dans le texte de Derrida «La main de Heidegger (Geschlecht II) ", où ce dernier le cite à partir de cette même section d'Être et Temps (qui, pour lui, «scande de façon saisissante cette longue manœuvre qui fait du chemin de pensée et de la question du sens de l'être une longue et continue méditation de la main $\left.{ }^{15} »\right)$ avant de passer à la dénonciation par Heidegger de la machine à écrire dans son séminaire sur Parménide (1942-

13. Derrida, «Entre le corps écrivant et l'écriture...", p. 63. Notre traduction anglaise de ce texte sera d'ailleurs bientôt publiée dans un livre intitulé Thinking What Comes édité par Geoffrey Bennington et Kas Saghafi.

14. Martin Heidegger, Être et Temps, trad. fr. François Vezin, Paris, Gallimard, 1986, p. 104; Sein und Zeit, dans Gesamtangabe, II, Francfort-sur-le-Main, Vittorio Klostermann, 1977, p. 92.

15. Derrida, "La main de Heidegger (Geschlecht II)», dans Psyché II. Invention de l'autre, nouvelle édition revue et augmentée, Paris, Galilée, 2003, p. 52. Remarquons qu'un important passage de cet essai sur la machine à écrire et le Parménide de Heidegger (ibid., 
1943). Dans ce texte tardif, nous retrouvons en effet Heidegger alors qu'il condamne la machine à écrire parce qu'elle «dévaste» et «dégrade» le mot, parce qu'elle "arrache l'écriture au domaine d'essence de la main, c'est-àdire de la parole ${ }^{16}$. Si pour Nietzsche la machine à écrire devient une collaboratrice active de la production de la pensée (le verbe qu'il utilise est bien "mitarbeiten", suggérant un travail conjoint ou un co-labeur dans la pensée), pour Heidegger il ne peut pas y avoir de pensée lorsqu'il n'y a pas d'authentique Handwerk. La machine à écrire ne peut que disperser le logos — et l'être de l'humain — dans un "nuage que rien n'annonce ${ }^{17}{ }^{\text {». Comme }}$ le fait remarquer Derrida, Heidegger privilégie le Handschrift (la "manuscripture » traduit Derrida) aux dépens de la mécanisation. La copie «tapée », le tapuscrit, repose sur une alliance essentielle entre la main, la parole et le logos, et plus encore sur une conception de cette alliance comme le propre de l'humain. Cet «humanisme métaphysique" signale déjà l'importance de la machine à écrire pour la question du geschlecht et de la lecture de Heidegger que fera Derrida dans Geschlecht $I I I^{18}$. La machine à écrire ne menace pas seulement l'intégrité et la spécificité d'un geschlecht humain qui, selon Heidegger, résiderait dans la main — et en particulier dans l'écriture manuscrite - , elle induit aussi, du même coup, une dissémination qui atteint directement le motif essentiel du rassemblement (Versammlung), comme le suggère Derrida, dans la lecture heideggérienne de Trakl. «La mécanisation typographique détruit cette unité du mot, cette identité intégrale, cette intégrité propre du mot parlé que la manuscripture, à la fois parce qu'elle paraît plus proche de la voix ou du corps propre et parce qu'elle lie les lettres, préserve et rassemble ${ }^{19}$." Derrida va plus loin quant à l'effet de rassemblement de l'écriture manuscrite en citant Heidegger: «l'écriture [est], dans sa provenance essentielle, manuscripture (Die Schrift ist in ibrer Wesensherkunft die Hand-schrift ${ }^{20}{ }^{\prime}$. Derrida étend ensuite son analyse jusqu'à révéler, aussi contre-intuitif qu'il y parait, le phonocentrisme que sous-tend ce privilège heideggérien de l'écriture manuscrite:

Et j'ajouterai, ce que Heidegger ne dit pas mais qui me paraît encore plus décisif, manuscripture immédiatement liée à la parole, c'est-à-dire plus vraisemblablement système d'écriture phonétique, à moins que ce qui rassemble Wort, zeigen et Zeichen ne passe pas toujours nécessairement par la voix et

p. 53-54) n'apparaît que dans la version publiée de ce texte et non dans le passage correspondant de son séminaire "Le fantôme de l'autre» de 1984-1985 («Septième séance », ff. 4).

16. Heidegger, Parménide, trad. fr. Thomas Piel, Paris, Gallimard, 2011, p. 133.

17. Ibid., p. 140: «La machine à écrire est un nuage que rien n'annonce, c'est-à-dire un cèlement qui, malgré sa présence insistante, se retire et à travers lequel le rapport de l'être à l'homme subit une mutation.»

18. Derrida, Geschlecht III. Sexe, race, nation, humanité, Geoffrey Bennington, Katie Chenoweth et Rodrigo Therezo (éds), Paris, Éditions du Seuil, 2018.

19. Derrida, «La main de Heidegger (Geschlecht II)», p. 53.

20. Ibid., p. 54. 
que la parole dont parle ici Heidegger soit essentiellement distincte de toute phonè. La distinction serait assez insolite pour mériter d'être soulignée. Or Heidegger n'en souffle pas mot. Il insiste au contraire sur la co-appartenance essentielle et originaire de Sein, Wort, legein, logos, Lese, Schrift comme Hand-schrift. Cette co-appartenance qui les rassemble tient d'ailleurs au mouvement de rassemblement même que Heidegger lit toujours, ici comme ailleurs, dans le legein et dans le Lesen ("... das Lesen d.h. Sammeln...»). Ce motif du rassemblement (Versammlung) commande la méditation du Geschlecht dans le texte sur Trakl que j'évoquerai brièvement tout à l'heure ${ }^{21}$.

Dans la pointe du stylo qui rassemble être et $\log o s$, donc, nous pouvons déjà voir la «pointe de la lance ${ }^{22}$ » qui rassemble le site du poème et gouverne la pensée de Heidegger sur l'unique et indivisible Ort dans son essai sur Trakl.

Si nous examinons maintenant attentivement ce passage du tapuscrit du séminaire de 1984-1985, nous voyons qu'au moment même où Derrida est en train de citer Heidegger affirmant que «l'écriture [est], dans sa provenance essentielle, manuscripture ", il frappe la mauvaise touche, et ce, à répétition - écrivant fautivement les mots "écriture » et "manuscripture» eux-mêmes à de multiples reprises avant de finalement les corriger, créant du même coup une surimpression des lettres $c, u, t$, et $p$. Une sorte de collaboration entre la main et la machine se fait alors remarquer, allant à l'encontre de cette pensée du rassemblement dans l'écriture - et tout ce que cela implique pour le geschlecht et la main -, marquant au contraire l'erreur et l'errance dans l'écriture de l'écriture et sa soi-disant "essence ». L'idée heideggérienne de l'écriture comme écriture manuscrite, indissociable de celle de la pensée comme manuscripture, avec laquelle elle va main dans la main, est en quelque sorte re-marquée ici dans le tapuscrit de Derrida, souligné concrètement, voire corporellement, par une pensée typographique que nous pourrions aussi appeler du nom de «déconstruction». En un certain sens, la rencontre entre Derrida et Heidegger dans la série des Geschlechter, le croisement de leur signature, la lecture derridienne de Heidegger qu'il décrit précisément comme une "surimpression ${ }^{23}$ ", est peut-être d'abord et avant tout la rencontre entre deux régimes médiatiques ou deux protocoles, deux ensembles de Schreibzeng, c'est-à-dire deux types de mains. Or il n'y a que l'une de ces deux mains qui peut faire des fautes de frappe, et l'une des deux seulement peut penser la faute de frappe à l'œuvre dans Geschlecht.

À ce point, nous pardonnerions à la lectrice et au lecteur les observations méthodologiques qu'elle ou il pourrait bien nous objecter, aussi bien que toutes ses questions qui iraient dans le sens de celles-ci: peut-on vraiment lire les fautes de frappe de Derrida? Peut-on vraiment les prendre au sérieux? Nous savons que Derrida était un mauvais dactylographe, mais

21. Ibid.

22. J. Derrida, Geschlecht III, p. 47.

23. Ibid., p. 41, note 1 . 
encore ? Plusieurs personnes - peut-être même plusieurs philosophes — ont elles aussi été de terribles dactylographes. Mais qu'est-ce que ces fautes de frappe peuvent bien avoir à faire avec la pensée de Derrida, avec sa philosophie, voire avec la philosophie, avec Geschlecht et toutes ces questions autrement plus sérieuses que nous pourrions aborder avec ces textes? On pourrait difficilement remettre en doute la légitimité de ces questions, mais je demanderais néanmoins à cette lectrice ou ce lecteur de considérer au moins ce qu'elle ou il présuppose déjà de l'écriture, et si ce type d'objections - qui repose implicitement ou explicitement sur une conception particulière de l'écriture voulant qu'elle soit secondaire et représentative, externe à la pensée et au logos — n'a pas déjà, justement, été fortement ébranlé et mis à mal par le travail de Jacques Derrida dès De la grammatologie? En ce sens, nous aurions aussi tout avantage à revisiter le séminaire La vie la mort de 1975-1976, récemment publié, dans lequel Derrida porte une attention considérable - ce que nous pourrions appeler une attention sérieuse, sinon une lecture au sens fort de ce terme - a une toute petite coquille dans l'édition française du Nietzsche de Heidegger où un espace avait été laissé à la place du « $\mathrm{z}$ » dans le nom du philosophe ( $N$ Niet sche $\left.{ }^{24} »\right)$. Je reviendrai d'ailleurs plus loin sur une autre coquille, non moins étonnante, à laquelle s'intéressera plus tard Derrida, cette fois-là dans sa propre œuvre. Tout cela pour dire que, d'un point de vue méthodologique, la faute de frappe se présente non seulement comme un objet légitime de lecture déconstructive, mais aussi, effectivement, comme une stratégie ou le site fécond d'une telle lecture, qui frappe au cœur de ce qu'une «lecture déconstructive» devrait être.

Si les fautes de frappe s'imposent d'elles-mêmes dans la série des Geschlechter, ce n'est pas seulement en raison de la prise en considération, par Derrida, du rejet heideggérien de la machine à écrire, mais aussi et plus impérativement en raison de ce qui se joue dans ce mot ou dans cette marque, ce geschlecht. "Geschlecht, le mot ou la marque ${ }^{25}$ ", Derrida ne cesse de répéter cette formulation dans son séminaire, marquant par ce mouvement la difficulté, voire l'impossibilité, de parler du geschlecht comme d'un «référent déterminable et unifiable ${ }^{26} »$, il est en quelque sorte sans référent audelà du mot ou de la marque. C'est particulièrement le cas chez Heidegger et son usage du mot allemand "geschlecht» que Derrida traque minutieusement dans sa série de quatre essais: "Car c'est la composition et la décomposition de cette marque, le travail de Heidegger dans sa langue, son écriture manuelle et artisanale, son Hand-Werk que les traductions existantes (fran-

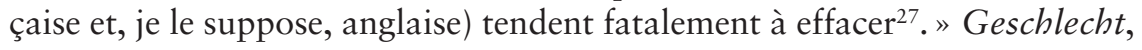

24. Voir Derrida, La vie la mort. Séminaire (1975-1976), Pascale-Anne Brault et Peggy Kamuf (éds), Paris, Seuil, 2019, p. 205 sq.

25. Derrida, Geschlecht III, p. 36, note 1.

26. Derrida, «La main de Heidegger (Geschlecht II)», p. 58.

27. Derrida, Geschlecht III, p. 36. 
bien sûr, n'est pas n'importe quel mot ou n'importe quelle marque: c'est le mot pour «type» qui porte en lui-même le Schlag, le coup, la frappe, l'empreinte.

Dans Geschlecht III, Derrida nous rappelle ainsi, sans relâche, que la «typographie, l'empreinte et l'impression graphiques", ce qui "écrit en frappant ", font partie de la " "famille" lexico-sémantique ${ }^{28}$ du geschlecht (auquel en outre le mot "famille" appartient lui-même). Comme l'écrit Rodrigo Therezo dans sa préface à Geschlecht III, "la frappe, le coup ou l'empreinte (Schlag) est ce qui fait d'un Geschlecht un Geschlecht pour Heidegger - qui déplace le sens de Geschlecht vers sa famille étymologique (gesleht et gislahti sont les formes collectives du vieil allemand slaht pour Schlag $\left.{ }^{29}\right)$ ". Geschlecht est un mot qui, comme le suggère Therezo, "re-marque en lui-même la marque ${ }^{30} »$ ou, pourrions-nous ajouter, refrappe la frappe.

Pour Heidegger dans son essai sur Trakl, il y a en effet une «bonne frappe $^{31}$ ", une frappe juste, un "rechte schlag ${ }^{32}$ ", qui marque chaque geschlecht le constituant ainsi en tant que tel. Pas de geschlecht sans schlag. Les ennuis commencent cependant pour Heidegger avec la «mauvaise frappe ${ }^{33}$ ", la coquille ou la faute - qui affecterait depuis l'extérieur le geschlecht comme tel. C'est ce second coup qui introduit la "guerre des sexes ${ }^{34}$ », cette violente discorde ou dissension qui, toujours selon Heidegger, mène à une séparation effrénée, ce que Derrida appelle, lui, une "mauvaise dissémination $^{35}$ ». À la dixième séance du séminaire «Le fantôme de l'autre» reprise dans Geschlecht III, Derrida déplace cette question de la «bonne» et de la «mauvaise» frappe vers celle d'une «bonne» et d'une «mauvaise» écriture qui fait de l'entreprise heideggérienne un geste "plus platonicien et plus chrétien qu'il n’y paraît », ce «juste coup » ou cette "juste frappe » ${ }^{36}$ du geschlecht s'accorderait donc implicitement, chez Heidegger, avec l'écriture manuelle et le rassemblement qu'implique ce travail de la main. La «juste frappe » du geschlecht n'exclurait ainsi pas seulement l'erreur ou l'errance disséminale de la faute de frappe, il exclurait $d u$ coup et depuis l'origine toute frappe mécanique, toute frappe typographique. En effet, pour Heidegger, la machine à écrire, en un sens, ne peut que produire des fautes

\section{Ibid., p. 74.}

29. Rodrigo Therezo, «Préface», dans Derrida, Geschlecht III, p. 17.

30. Ibid., p. 11.

31. Heidegger, «La parole dans l'élément du poème. Situation du Dict de Georg Trakl », dans Acheminement vers la parole, trad. fr. Jean Beaufret, Wolfgang Brokmeier et François Fédier, Paris, Gallimard, p. 53.

32. Heidegger, "Die Sprache im Gedicht, Eine Erörterung von Georg Trakls Gedicht" [1959], dans Unterwegs zur Sprache, Friedrich-Wilhelm von Hermann (éd.), Francfort-sur-leMain, Vittorio Klostermann, 1985, p. 46.

33. Derrida, Geschlecht III, p. 74.

34. Ibid., p. 147. Voir aussi Derrida, "Chorégraphies", dans Points de suspension. Entretiens, Elizabeth Weber (éd.), Paris, Éditions Galilée, 1992, p. 106.

35. Derrida, Geschlecht III, p. 97.

36. Ibid., p. 74. 
de frappe ou des «mauvais» coups; toute erreur typographique serait le symptôme de cette errance essentielle, la mauvaise dissémination forgée par la machine à écrire. Comme Derrida le soulève par ailleurs, cela présuppose en filigrane un schéma relationnel particulier entre essence et accident, par exemple de la division qui affecterait de l'extérieur l'indivisibilité essentielle du point ou du lieu, du site, de la "pointe de la lance» comme telle. Derrida insiste par conséquent sur l'idée que l'accident n'est possible que si l'essence est déjà affectée, d'une certaine manière, depuis l'origine. "Si l'essence est accidentable, elle est a priori accidentée ${ }^{37}$ », propose-t-il en une formulation laconique elle-même marquée, dès le tapuscrit du séminaire, par un accident typographique (le mot «accidentable " étant écrit «accidentaboe» avant d'être corrigé par un «l» surimposé au « $\mathrm{O}$ » fautif).

De cette manière, nous pouvons commencer à comprendre la pensée derridienne du geschlecht en tant qu'elle se produit par et avec la machine à écrire, la faute de frappe, le lettrage, une pensée de l'empreinte frappée, du coup sur le support papier. Je formulerai donc ainsi ma propre hypothèse, laquelle se tient au croisement de deux langages: il n'y aurait pas de $\tau \dot{\pi} \pi \mathrm{s}$ (typos) sans faute de frappe (typos). Et nous ne saurions assez insister, ici, sur le fait qu'il faut s'avancer sur ce terrain avec prudence pour ne pas simplement opposer l'écriture manuelle et l'écriture typographique dans un geste qui capitaliserait sur une métaphysique technomédiatique qu'il ne ferait en définitive que reconduire. Derrida s'évertue en effet dans "Geschlecht II » à parler d'écriture manuscrite comme "manuscripture ", car "ne l'oublions pas comme on le fait si souvent, l'écriture de la machine à écrire contre laquelle Heidegger va élever un réquisitoire implacable est aussi une écriture manuelle ${ }^{38} »$. De plus, comme il le suggère aussi dans une entrevue publiée dans Papier Machine sous le titre "La machine à traitement de texte ", nous ne pouvons pas penser l'écriture manuscrite comme un geste prétechnologique ou prémécanique: "quand on écrit “à la main", on n'est pas à la veille de la technique, il y a déjà de l'instrumentalité, de la reproduction régulière, de l'itérabilité mécanique ${ }^{39}$ ». Quand nous "tapons" sur un clavier, nous sommes toujours en train d'écrire "à la main », mais selon un autre régime, une autre économie corporelle, une autre configuration technique de la main et de la langue. Et pourtant, quelque chose arrive à la pensée, comme le suggère Nietzsche, pour peu que nous la laissions arriver, que nous répondions à l'appel de cette nouvelle collaboration, que nous laissions venir la faute (typographique).

37. Ibid., p. 106.

38. Derrida, «La main de Heidegger (Geschlecht II)», p. 53.

39. Derrida, "La machine à traitement de texte", dans Papier Machine. Le ruban de machine à écrire et autres réponses, Paris, Galilée, 2001, p. 152. 
Dans l'entrevue de 2001 où il admet faire «beaucoup de fautes ${ }^{40}$ ", il n'y a pas que de l'hyperbole et de l'autodérision. Si nous prenons un exemple de dix lignes tirées du tapuscrit du séminaire de 1984-1985 — tel qu'on peut le consulter dans les archives du philosophe à UC Irvine - et plus particulièrement le passage où Derrida cite le $\mathbb{S} 15 \mathrm{~d}$ 'Être et Temps, nous comptons au moins vingt-quatre erreurs typographiques, essentiellement des fautes de frappe, qui ont été repérées dans la majorité des cas par l'auteur. Alors, le dactylo s'arrête, revient et se corrige, créant une impression presque constante de redoublement et de surimpression, une retouche de la touche dans le tapuscrit. Derrida, d'ailleurs, ne cache pas avoir d'abord appris à taper en 1956 aux États-Unis: "J'ai appris à taper, vite et mal, à Cambridge et Harvard, où j'ai passé un an après mon temps d'école ${ }^{41}$. "Contrairement à Nietzsche, Derrida ne fut pas un «early adopter» ou un utilisateur enthousiaste de la machine à écrire; après tout, il écrivit ses premiers livres incluant De la grammatologie - à la main, ne les tapant qu'en dernière instance, au moment de produire les versions finales ${ }^{42}$. «Ma première machine à écrire, je l'ai achetée aux États-Unis. Il fallait que je tape ${ }^{43}$ ", se confie-t-il, comme si taper lui manquait, comme si la nécessité de la faute de frappe se faisait sentir. Ce fut donc à Cambridge (É.-U.) que Derrida acheta sa première machine à écrire qu'il décrit à de nombreux endroits comme étant une «petite Olivetti» avec un «clavier international ${ }^{44}$. Il se rappelle plus précisément, du moins dans cette entrevue, que son Olivetti était une "Lettera 32 », mais ce souvenir s'avère être, en fait, quelque chose comme une faute de frappe dans l'anamnèse. Effectivement, la Olivetti 32 ne fut commercialisée qu'au milieu des années 1960 (Derrida n'a donc pas pu s'en procurer une en 1956), et c'est en fait le modèle 22 qui avait le "clavier international» dont parle Derrida et qu'il se procura parce qu'il n'y avait pas de clavier français aux États-Unis. Cela n'empêche pas cette machine à écrire

40. Derrida, «Entre le corps écrivant et l'écriture...», p. 63.

41. Ibid., p. 63.

42. Voir à ce sujet Derrida, «La machine à traitement de texte», p. 152-153: «Pour les textes qui m'importaient, ceux que j'avais le sentiment un peu religieux "d'écrire”, je bannissais même le stylo. Je trempais dans l'encre un long porte-plume, la pointe légèrement courbée d'une certaine plume à dessin, en multipliant brouillons et versions préliminaires avant de les arrêter sur ma première petite Olivetti à clavier international que j'avais achetée à l'étranger. Je l'ai encore. Je devais avoir l'impression que mon artisanat d'écriture frayait en effet sa voie dans cet espace de résistance, au plus près de cette main de la pensée ou du mot auquel nous rappellent ces pages de Heidegger que j'ai tenté plus tard d'interpréter dans "La main de Heidegger". Comme si cette liturgie pour une seule main était requise, comme si cette figure du corps propre ramassé, penché, appliqué et tendu vers une pointe encrée était aussi nécessaire au rituel d'une gravure pensante que la surface blanche du subjectile de papier sur le support de la table. Mais je ne me cachais jamais que, comme dans tout cérémonial, il devait y avoir de la répétition, et déjà une espèce de mécanisation. »

43. Ibid. Nous soulignons.

44. Ibid. 
portative d'avoir été son modèle de prédilection pendant plus de deux décennies:

J'ai acheté une petite Olivetti 32 avec un clavier international, parce que je n'ai pas trouvé de clavier français là-bas. Ce qui fait que pendant de longues années, de 1957 jusqu'aux années soixante-dix, je devais profiter de voyages aux États-Unis pour retrouver ce clavier international auquel j'étais habitué. Je n'ai pas pu taper sur un clavier français jusqu'à cette date-là. Chaque fois, je remplaçais une petite Olivetti par une petite Olivetti ${ }^{45}$.

Vers la fin des années 1960, Derrida rapporte qu'il a commencé à utiliser la machine à écrire non plus pour copier les textes qu'il avait déjà rédigés à la main, mais pour y écrire directement, composant d'emblée ses brouillons de séminaires et de livres sur celle-ci. En 1979, Derrida changea sa machine à écrire mécanique pour une version électrique, dès l'écriture de La Carte postale qui l'accompagna dans ses premiers pas avec cette nouvelle technologie. C'est sur cette dernière que la série des Geschlechter naquit, sur cette machine électrique que les trois premiers morceaux furent tapés, "très vite, très mal, avec beaucoup de fautes ".

Il ne faudrait cependant pas en déduire pour autant que Derrida est négligent à l'égard de ses textes, loin de là. En fait, plusieurs documents tirés de ses archives révèlent une précision typographique des plus exigeantes, voire "obsessive", en ce qui concerne la publication et la révision de ses textes. Les exemplaires de ses propres livres, tels que nous pouvons les consulter à la bibliothèque de Princeton, regorgent par exemple de traces de relectures attentives et de corrections (mais pas toujours) en vue d'une éventuelle édition. En ce sens, nous trouvons à l'intérieur de sa copie de la première édition de De la grammatologie une feuille de papier blanc ${ }^{46}$ contenant une liste de dix corrections à faire en vue de la seconde édition (par exemple, à la page 290: «C'est le salut [statut] du signe qui se trouve ainsi marqué par la même ambiguité»). L'utilisation d'encres et de crayons de couleurs différentes nous indique aussi que ces corrections - par ailleurs systématiquement rapportées dans le corps du texte aux pages correspondantes ont été compilées sur une longue période de temps et au gré de relectures successives. Pliée et insérée dans les pages de ce livre, nous retrouvons en outre une lettre dactylographiée par Samuel Weber et datée du 19 avril 1969, envoyée de Berlin. Dans cette lettre, l'ami de Derrida l'alerte quant à la présence de «plus de vingt fautes d'impression» qu'il a répertoriées dans cette

45. Ibid.

46. Il est possible de consulter une photographie de cette image, de même que toutes les annotations marginales de Jacques Derrida dans son édition de De la grammatologie, grâce à l'outil de recherche en ligne, Derrida's Margins, entièrement consacré aux annotations dans la bibliothèque de Jacques Derrida hébergée à l'Université de Princeton [en ligne: https://derridasmargins.princeton.edu/library/derrida-de-la-grammatologie-1967/gallery/pp-388-389-insertiond-recto/]; consulté le 30 octobre 2019]. 
édition. Celles-ci s'ajoutant à celles que Derrida lui avait apparemment déjà signalées.

Ce qui est plus frappant encore que cette volonté toute derridienne de ne pas céder à l'erreur ou à l'errance, c'est une autre faute de frappe impliquée dans $\mathrm{Da}$ la grammatologie, une coquille maintenant invisible que Derrida a cependant longuement commentée dans son essai "Le ruban de machine à écrire. Limited Ink II ». Cette dernière met en lumière une dimension moins évidente de la faute de frappe, à savoir sa singularité archivistique, voire sa jouissance archivistique. La coquille en question concerne l'exergue tiré des Confessions de Rousseau qui ouvre la seconde partie de De la grammatologie: "J'étais comme si j'avais commis un inceste ${ }^{47}$." Dans "Le ruban de machine à écrire », Derrida rappelle qu'au moment où il relisait les Confessions en préparant la première année du séminaire Le parjure et le pardon, "on venait d'exhumer puis de déchiffrer, en Picardie, une archive prodigieuse ${ }^{48} »$. L'archive en question était constituée d'un insecte pris dans l'ambre - surpris par la mort, capturé et archivé intact - au moment même où il suçait le sang d'un autre insecte, quelque 54 millions d'années avant l'apparition de l'humain sur la Terre. Voici ce que Derrida dit au sujet de cette remarquable archive animale:

Une chose est de connaître des sédiments, des pierres, des végétaux qu'on peut dater de ce temps sans temps où rien d'humain ni même de vivant ne faisait signe sur la terre. C'est une autre chose que de se référer à un événement singulier, à ce qui eut lieu une fois, une seule fois, en un instant non répétable, comme cet animal surpris par la catastrophe au moment, à tel instant, à telle pointe stigmatique du temps où il était en train de jouir à sucer le sang d'un autre animal [...]. Car on parle aussi de deux moucherons immobilisés dans le même ambre couleur de miel quand ils furent surpris par la mort en train de faire l'amour: cinquante-quatre millions d'années avant l'apparition de l'homme sur la terre, une jouissance eut lieu dont nous gardons l'archive. Cela nous arrive encore, cela arrive encore jusqu'à nous. Nous avons là, déposée, consignée sur un support, protégée par le corps d'un cercueil d'ambre, la trace ellemême corporelle d'un événement qui n'eut lieu qu'une fois ${ }^{49}$.

Ce moment précis d'une jouissance archivée de l'insecte précède les humains et dépasse de plusieurs millions d'années leurs appareils archivistiques: c'est littéralement l'archive d'un autre Geschlecht. Quand nous tombons ainsi par hasard sur cette archive singulière — tout comme Derrida était lui-même tombé par hasard sur cette découverte dans un journal dont la coupure de

47. Jacques Derrida, De la grammatologie, Paris, Les Éditions de Minuit, 1967 p. 143.

48. Derrida, "Le ruban de machine à écrire. Limited Ink II", dans Papier Machine, p. 107. Cet essai est lui-même partiellement tiré de la «Dixième séance» du séminaire (19971998) où il est aussi question de cette étrange faute. Voir Derrida, Le parjure et le pardon. Séminaire Volume i (1997-1998), Ginette Michaud et Nicholas Cotton (éds), Paris, Éditions du Seuil, 2019, p. 371-374.

49. Ibid., p. 108; Le parjure et le pardon I, p. 371-372. 
presse est toujours intégrée aux pages du tapuscrit de son séminaire conservé dans les voûtes de l'Institut Mémoires de l'édition contemporaine (IMEC) - nous sommes aussi surpris que lui par cette archive qui se donne ellemême à lire au moins autant qu'elle demeure en quelque sorte illisible, secrète, à plus d'un titre.

Si nous nous attachons à ce passage du séminaire qui pourrait paraître anecdotique, c'est parce que quelques pages plus loin, Derrida le rattache explicitement aux questions de la coquille typographique et de la faute transgressive:

Je ne savais pas, il y a un instant, pourquoi je vous raconte ces histoires d'archives: archives d'insecte vampire, archives d'animaux en train de faire l'amour il y a bien plus de cinquante-quatre millions d'années - et archives comme Confessions. Si je crois me rappeler maintenant, bien que ce fût d'abord inconscient et ne me revient qu'après coup, c'est que tout à l'heure, je vais parler d'effacement et de prothèses, des falsifications de la lettre, de la mutilation des textes, de corps d'écriture [...]. Or, et là il faut me croire, parce que je vous dis la vérité, comme toujours, quand j'ai cité Rousseau, dans De la grammatologie, en 1967, et quand, à titre d'exergue pour toute la partie (presque tout le livre) que je consacrai alors à Rousseau, j'ai rappelé ses mots, "J'étois comme si j'avais commis l'inceste ", eh bien, les premières épreuves du livre me sont revenues avec une étrange coquille. J'avais été tenté, dans un premier temps, de ne pas la corriger. L'imprimeur en effet avait écrit: «J'étois comme si j'avais commis l'insecte.» Coquille peut-être destinée à se garder de l'inceste, mais à en protéger qui ou quoi ? Anagramme parfait (insecte/inceste) que, pour respecter la machine grammaticale, j'ai dû me résoudre à rectifier et à normaliser. Je suis ainsi revenu de l'insecte à l'inceste, refaisant tout le chemin, les cinquante-quatre millions d'années qui mènent de l'animal suceur de sang au premier homme des Confessions $[\ldots]^{50}$.

Dans cette scène, l'erreur typographique en question - la "coquille ", un mot qui appartient aussi au champ lexical de l'animalité (shell, la carapace, le coquillage, la carcasse, etc.) — révèle en quelque sorte ce qui est à l'œuvre inconsciemment dans le texte. Comme moment de jouissance, elle le rappelle et l'exhume, à l'instar de ces insectes pris dans l'ambre. Surtout, cette coquille séduit ( "J'avais été tenté, dans un premier temps, de ne pas la corriger $^{51 »)}$ tout en devant, à la fin, être normalisée, standardisée, "pour respecter la machine grammaticale». Derrida insiste sur l'idée que la faute, ici, fut introduite non pas en raison de sa (piètre) qualité de dactylographe, mais par le compositeur lors de la préparation des épreuves. Cette substitution typographique qui fait de l'inceste un insecte apparaît aussi comme une protection ("se garder», "protéger»). C'est une faute (de frappe) destinée à protéger d'une autre faute, à savoir du péché inavouable de l'inceste. Et

50. Ibid., p. 110-111. Le parjure et le pardon I, p. 373-374.

51. Ibid. Nous soulignons. 
n'oublions pas que ladite composition des textes, à partir de l'introduction en typographie des linotypes et des monotypes, se fait à l'aide d'un clavier qui ressemble en tout point à celui de la machine à écrire, si bien que cette erreur du typographe, en 1967, consiste nécessairement en une mauvaise frappe sur les touches! Mais ce n'est pas tout. Il convient de noter ici qu'une autre faute de frappe subsiste dans le tapuscrit du séminaire Le parjure et le pardon, et cette fois-ci elle est bien de Derrida lui-même. Tenace, elle est même reportée dans les versions publiées en français et en anglais du texte «Le ruban de machine à écrire». Le premier verbe de cette citation, "J'étois", est en effet écrit par Rousseau dans son ancienne orthographe (où la terminaison pour l'imparfait s'écrivait -ois), alors que le second verbe " avoir ", auxiliaire attaché à l'inceste lui-même — a été pour sa part modernisé (sa terminaison est bien en -ais), mais ce n'était pas le cas dans l'édition de référence que consulte Derrida: "J'étois comme si j'avois commis un inceste ${ }^{52}$ » devient "J'étois comme si j'avais commis l'inceste ». Tout se passe comme si le premier verbe était demeuré prisonnier dans l'ambre de l'orthographe du XVIII ${ }^{e}$ siècle, à l'image de l'insecte pris en train de sucer du sang ou de faire l'amour, alors que le second verbe a été normalisé — ramené au goût du jour - nous pourrions dire refoulé. Il conviendrait aussi de noter que le mot choisi par Derrida pour parler de l'erreur dans ce contexte est «coquille» et que celui-ci évoque non seulement une certaine animalité comme nous le disions plus haut, mais a fortiori une animalité qui se protège à l'aide d'une coque, dure, d'une enveloppe protectrice, qui s'inversant ou se retournant sur elle-même (l'inversion et le retournement étant l'une des étymologies proposées pour l'utilisation de ce mot qui signifie aussi la faute de frappe et l'erreur d'impression). Cette faute, qui couvre ou protège d'une autre faute, nous mène directement à une autre scène de Geschlecht dans laquelle les nombreuses significations de ce mot semblent se replier ou se retourner les unes sur les autres - différence sexuelle, famille, génération, lignée, clan, maison, espèce, type, etc. - , et ce, d'une manière interdite, pécheresse, transgressive, coupable, fausse, fautive ou faulty, à coup sûr impardonnable. La faute de l'inceste hante effectivement Geschlecht III à plus d'un titre, notamment en ce qui a trait à la relation incestueuse entre Georg Trakl et sa sœur Grete, laquelle fait l'objet de plusieurs allusions dans ses poèmes et semble marquer presque autant la pensée heideggérienne du "tendre dédoublement ${ }^{53}$ ", innocent et bon, de la différence sexuelle comme relation entre les Geschlechter, entre frère et sœur: une frappe sans faute.

52. Jean-Jacques Rousseau, Les Confessions, dans Euvres complètes, I, Bernard Gagnebin et Marcel Raymond (éds), avec, pour ce volume, la collaboration de Robert Osmont, Paris, Gallimard, 1959, p. 197. Nous soulignons.

p. 74 .

53. Heidegger, «La parole dans l'élément du poème », p. 80; "Die Sprache im Gedicht », 
Il est frappant de constater que le mot «inceste» ne figure nulle part dans Geschlecht III, ce texte que Derrida a annoncé plusieurs fois comme étant "à paraître" au cours de sa vie, mais qu'il n'a étrangement jamais entrepris de réviser pour autant. En effet, au meilleur de ma connaissance, il n'a prononcé ce texte qu'une seule fois au cours de son séminaire de 19841985, ne nous laissant ainsi que le tapuscrit de ces séances, truffé de fautes, encore intactes et préservées dans une archive singulière qui ne fut exhumée que plusieurs années plus tard à l'IMEC. Dans ses archives, les fautes de frappe de Derrida demeurent visibles et lisibles, elles n'ont pas encore été ni corrigées ni "normalisées». Si nous comparons par exemple ce tapuscrit avec celui du séminaire de 1997-1998 que j'évoquais plus haut sur Le parjure et le pardon, nous constatons aisément à quel point ce dernier présente relativement peu de fautes de frappe par rapport au premier. Il y a probablement une raison d'ordre technologique qui explique cette différence, mais nous ne pouvons que le déduire à partir de ce que nous savons du contexte matériel de production de ce séminaire. Ainsi, les cours de 1997-1998 n’ont pas été élaborés sur une machine à écrire mais bien sur un ordinateur, avec un logiciel de traitement de texte (Word Processor), et donc avec tous ces outils d'écriture (Schreibzeug) que nous utilisons maintenant sur une base régulière: la touche "supprimer ", la vérification orthographique, l'autocorrecteur, etc. Avec l'ordinateur, en un certain sens, il n'y a plus de faute de frappe possible, mais celui-ci ouvre plutôt à un autre type de fautes - les erreurs de saisies - comme Derrida nous le rappelle à la fin de Geschlecht III. Il ouvre donc à un nouveau régime de "normalisation ", pour reprendre encore l'expression de Derrida.

En fait, au moment de la conceptualisation de Geschlecht III, nous sommes déjà dans ce que nous pourrions appeler un "crépuscule» de la machine à écrire chez le philosophe. Derrida identifie en effet, si l'on se souvient bien, ce moment précis, 1984-1985, comme étant le début de sa «résistance» à l'ordinateur, et ce, même s'il ne «capitulera » finalement que deux ans plus tard. À l'automne 1987, alors qu'il commence la quatrième et dernière année du cycle «Nationalité et nationalisme philosophiques" (19841988) de son séminaire, il a déjà entrepris de taper le texte de ses cours sur son "petit Mac». Cette séquence du séminaire marquerait donc la fin de la machine à écrire pour Derrida et, par conséquent, la fin d'une certaine faute de frappe dans l'écriture et la pensée de celui-ci, un adieu aux pages criblées de fautes signifiantes. Par exemple, celle qui en compte plus de vingt-sept où Derrida commente justement, dans la phrase de Trakl «Ein Geschlecht », les effets de l'espacement typographique qui selon la convention allemande accentue le texte de manière idiomatique, non pas en utilisant l'italique mais bien en intercalant des espaces entre chaque lettre: "E $i$ n Geschlecht». Lorsque Heidegger cite cette phrase - un moment clé dans sa propre lecture de Trakl —, il renvoie en effet au «seul mot espacé [gesperrt] dans les 
poèmes de Trakl ${ }^{54}$ ». Pour Heidegger, ce mot «souligné » (betont) «recèle le ton fondamental [Grundton] à partir duquel le Dict du Poète demeure tacite. L'unité de cette race [Ein Geschlecht] surgit de la souche qui [...] appareille la dissension [Zwietracht] des genres dans l'unité candide [einfältig] d'un dédoublement plus tendre $[\text { Zwiefalt }]^{55}$.» Et pourtant, comme le fait remarquer Rodrigo Therezo, le texte de Heidegger efface l'espacement en le remplaçant par le mot "Ein» en italique, "un geste qui s'avère lui-même phonocentrique, puisque Heidegger entend implicitement l'accent mis sur le "Ein" (betont) comme indicateur du ton fondamental de la poésie de Trakl dans son ensemble ${ }^{56}$ ". Même si Heidegger semble attentif aux éléments typographiques dans le texte de Trakl — son usage de la ponctuation, le fait que "Ein» est le seul mot accentué, etc. - il manque, néglige, se ferme les yeux et se protège de l'espacement qui diviserait le «un» rassemblé du Ein Geschlecht. Il en résulte une pensée du Geschlecht allant dans le sens de son rejet de la machine à écrire, une pensée qui donc ne peut ou ne veut lire que la «bonne» frappe, jamais la «mauvaise».

Nous pourrions conclure en faisant le point sur un mot en particulier qui est sujet à une erreur typographique dans le manuscrit dactylographié du séminaire qui devriendra Geschlecht II et Geschlecht III. Ce mot, ou mieux cette marque, est celui-là même de "Geschlecht»: un mot que nous pourrions maintenant être tenté de traduire par «type», mais aussi par (faute de) frappe ou par typo (en anglais). Derrida le tape très mal, continuellement, de plusieurs façons différentes et chaque fois renouvelées, comme si les doigts qui ont appris à taper sur un clavier «universel international» ne pouvaient pas tout à fait apprendre ou s'adapter à la machine nationale, voire nationaliste, du Geschlecht, une marque qui devient souvent entre les mains de Derrida un "Geclecht» ou un «Geglecht». Parfois, Derrida cesse même de taper ce mot qui s'écrit alors simplement «G». Ce qui semble cependant remarquablement cohérent dans toutes ces fautes de frappe, c'est le lieu même de leur erreur: au centre du Geschlecht, à savoir au lieu même du Schlag, au cœur du Geschlecht (de son site), c'est-à-dire précisément là où Geschlecht porte la marque de l'empreinte ou de la frappe. C'est aussi ce que nous pourrions appeler le cœur idiomatique de ce mot particulièrement allemand, pas seulement comme le lit Heidegger parce qu'il appelle en sa

54. Ibid. Je suis ici Rodrigo Therezo qui traduit "gesperrt» en anglais par "spacedout». Voir R. Therezo, "When Silence Strikes: Derrida, Heidegger, Mallarmé », The Oxford Literary Review, vol. 40, n 2, 2018, p. 238-262. [Dans sa traduction française, Jean Beaufret traduit ce passage ainsi: "C'est, autant que je sache, le seul mot écrit en italique dans toute l'œuvre poétique de Trakl. » Heidegger, «La parole dans l'élément du poème », p. 79 (NdT)].

55. Ibid., p. 79-80.

56. Therezo, «When Silence Strikes: Derrida, Heidegger, Mallarmé», p. 240. Nous traduisons. 
souche le vieil haut-allemand, mais plutôt d'un point de vue typographique, dans le rassemblement de ces quatre consonnes s. c. h. l., idiomatiquement allemandes, qui agissent comme une sorte de marque (un Deutsch-mark) frappant ou imprimant le Geschlecht comme on le fait pour la monnaie coined dirions-nous en anglais - se protégeant du coup de tout fauxmonnayage, piégeant les doigts non allemands inaccoutumés à les taper. Comme si, pourrions-nous dire, l'idiomaticité du Geschlecht - son empreinte nationale, sa typo-graphie - se faisait d'abord et avant tout ressentir comme autant de fautes de frappe entre deux doigts, engagés dans un combat mutuel avec la machine à écrire, pensant avec elle, frappant au cœur du nationalisme philosophique.

Je donnerai le dernier mot à Nietzsche qui composa un petit poème sur sa machine à écrire en 1882. Sur sa machine... c'est-à-dire au double sens de cette préposition qui veut aussi bien dire "avec elle» que "la prenant pour sujet ». Nietzsche, donc, composa un petit poème sur sa machine à écrire et le tapuscrit de cette page, tel qu'on peut le voir dans les archives du philosophe, se présente en caractères romains et en lettres capitales. Sa sphère à écrire de Malling Hansen, produite en dehors de l'Allemagne, n'écrivait en effet que dans cette police de caractères qui, doit-on le rappeler, était considérée en Allemagne comme "moderne", internationale et non allemande jusqu'en 1941 - contrairement à des polices plus nationales et conservatrices comme le Blackletter ou le Frakturschrift —, c'est-à-dire lorsque le parti nazi détermina arbitrairement que le Fraktur était une écriture "juive» et qu'il imposa l'utilisation des caractères romains.

Le 16 février 1882, Nietzsche a donc tapé le poème suivant:

LA SPHÈRE ÉCRIVANTE EST UNE CHOSE COMME MOI: EN FER POURTANT FACILEMENT VRILLÉE EN VOYAGES

PATIENCE ET TACT EN GRANDE QUANTITÉ SONT REQUIS AINSI QUE DES DOIGTS FINS, POUR NOTRE UTILISATION ${ }^{57}$.

(Traduit de l'anglais par Nicholas Cotton)

57. Nietzsche, «Poème Malling Hansen », trad. fr. Frédérique Vargoz, cité dans Kittler, Gramophone, Film, Typewriter, p. 341. 